\title{
High resolution magnetic resonance imaging of atherosclerosis and the response to balloon angioplasty
}

\author{
R A Coulden, H Moss, M J Graves, D J Lomas, D S Appleton, P L Weissberg
}

\begin{abstract}
Objective-To explore the use of high resolution magnetic resonance imaging (MRI) of the popliteal artery in defining atheroscelerotic lesions and to monitor the remodelling response to balloon angioplasty.

Methods-Four patients (aged 49-67) with symptomatic discrete popliteal artery stenoses, as demonstrated by conventional angiography, underwent balloon angioplasty. MRI of the diseased vessel was performed before and one week, one month, three months, and six months after therapeutic balloon angioplasty. Cine phase contrast MRI was used to estimate blood flow just proximal to the lesion before and after angioplasty.

Results-In all patients the extent of the atherosclerotic plaque could be defined, such that even in segments of vessel which were angiographically "normal", atherosclerotic lesions with cross sectional areas ranging from $49 \%$ to $76 \%$ of potential lumen area were identified. Following angioplasty, plaque fissuring and local dissection were easily identified and serial changes in lumen diameter, blood flow. and lesion size could be documented.

Conclusions-High resolution MRI can define the extent of atherosclerotic plaque in the peripheral vasculature and demonstrate the changes that occur with remodelling and restenosis following angioplasty. As a safe, reproducible technique MRI is ideal for assessing plaque and monitoring intervention, but further technological developments will be needed if similar or better images are to be achieved in other vascular beds.

(Heart 2000;83:188-191)
\end{abstract}

Keywords: magnetic resonance imaging; atherosclerosis; angioplasty

Lumen stenosis, as demonstrated by contrast angiography, is central to the clinical diagnosis and management of atherosclerotic vascular disease. Recently, however, it has become clear that the outcome from atherosclerosis is determined more by plaque composition than size. ${ }^{1-3}$ Plaques with a large lipid core, thin fibrous cap, and high macrophage content are more prone to rupture than similar sized plaques containing few inflammatory cells and a thick, smooth muscle cell rich, fibrous cap. Small, angiographically invisible plaques may rupture to cause a clinical event, ${ }^{4}$ while large atherosclerotic lesions may grow "outwards" and produce little lumenal narrowing. ${ }^{5}$ The limitations of contrast angiography in predicting the natural history of atherosclerosis are well known but have been emphasised by recent trials of lipid reduction with HMG-Co A reductase inhibitors, the statins. These have shown little impact on the angiographic appearance of pre-existing atherosclerotic lesions, but a major reduction in the clinical events precipitated by plaque rupture. ${ }^{6}$

Better imaging is required and this must be non-invasive, repeatable, and capable of imaging the vessel wall, as well as being able to measure lumenal stenoses. Magnetic resonance imaging (MRI) has the potential to fulfil this role. Magnetic resonance angiography is already well established in clinical practice and MRI has been shown to discriminate different components of developing atherosclerotic lesions in animals ${ }^{78}$ and man. ${ }^{9}$ To date, however, there have been no longitudinal studies of MRI to define disease progression in man. In this study we report the use of high resolution $\mathrm{T} 1$ weighted spin echo and time of flight (TOF) magnetic resonance angiography to image symptomatic atherosclerotic lesions in the popliteal artery of four patients before and after therapeutic balloon angioplasty. We show that MRI can define the initial atherosclerotic lesion and can demonstrate sequential reactive changes in the vessel wall following angioplasty, as well as providing information on blood flow. Our study therefore shows that with appropriate development MRI should become an important part of vascular imaging in the future.

\section{Methods}

Four patients (aged 49-67 years) with symptomatic atherosclerotic stenosis of the popliteal artery were examined by high resolution magnetic resonance imaging before and after percutaneous angioplasty. Imaging was performed up to seven days before angioplasty and then at one week, one month, three months, and six months afterwards. All examinations were made with a $1.5 \mathrm{~T}$ clinical magnetic resonance imaging system (Signa (5.4, SR17), GE Medical Systems, Milwaukee, Wisconsin, USA) using a $7.5 \mathrm{~cm}$ surface coil placed behind the knee.

Following a series of pilot images to identify the popliteal artery, a cross sectional cardiac gated, TOF magnetic resonance angiogram was performed to allow accurate positioning of the high resolution images. A cine phase contrast acquisition placed a short distance 
cranial to the stenosis gave an estimate of volume flow and allowed calculation of optimum trigger delay for subsequent imaging. The arterial wall was imaged with a transaxial, cardiac gated, $\mathrm{T} 1$ weighted spin echo sequence with the following parameters: time of echo (TE) $21 \mathrm{~ms}$, slice thickness $3 \mathrm{~mm}$, gap $1.5 \mathrm{~mm}$, $8 \mathrm{~cm}$ field of view (FOV), $512 \times 256$ matrix, number of excitations $(\mathrm{NEX})=2$, receiver bandwidth $8 \mathrm{kHz}{ }^{10}$ Superior, inferior, and anterior spatial presaturation was applied to
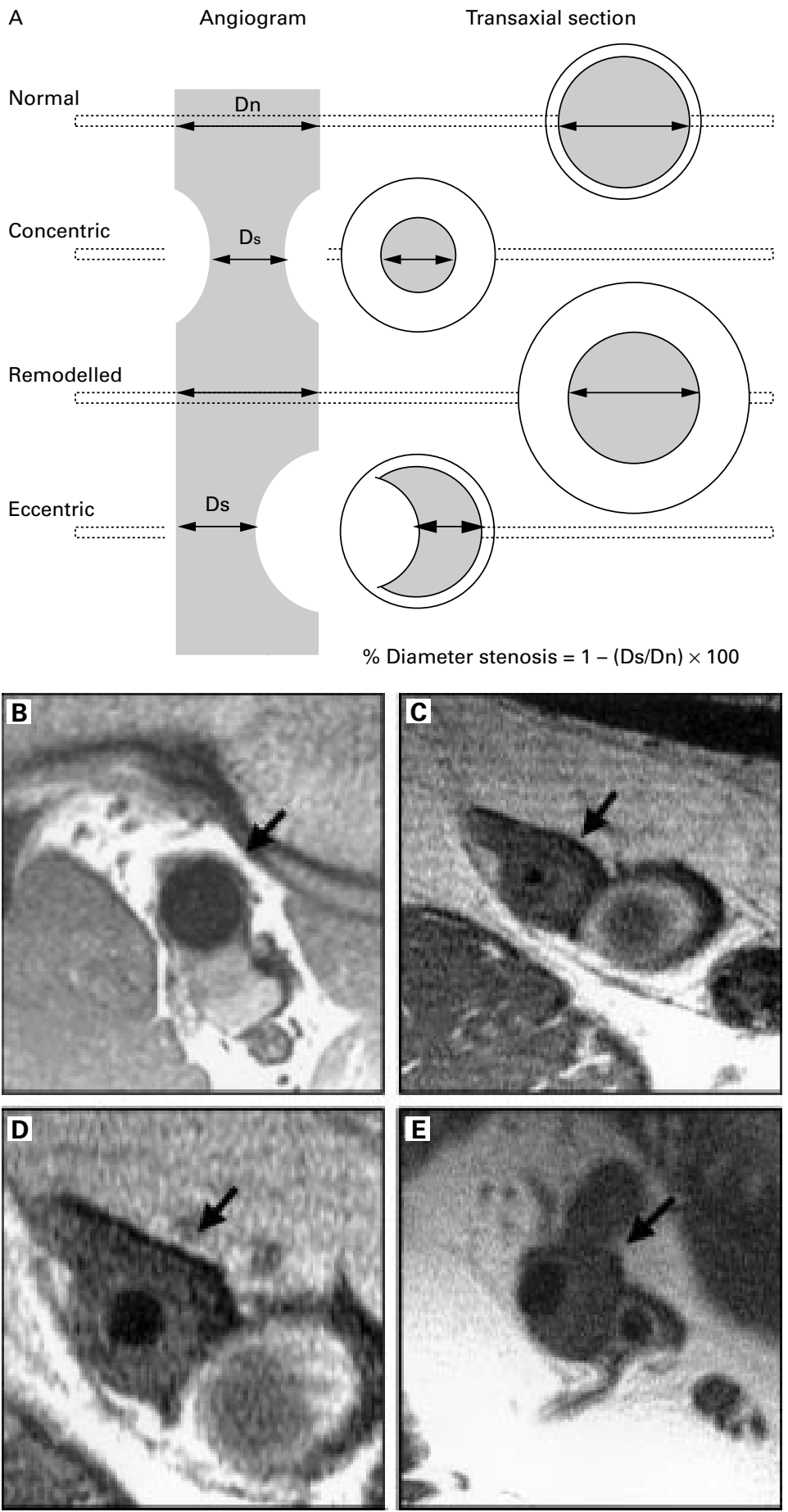

Figure 1 (A) Diagrammatic representation of a conventional angiogram with cross sections through the vessel wall. Cross sections are compared with high resolution magnetic resonance images of the popliteal artery (arrows) taken through a region of normal vessel (B), concentric plaque (C), remodelling (D), and eccentric plaque (E). The vessel adjacent to the artery is the popliteal vein and can be of variable size. eliminate slow flow artefact and aliasing resulting from the small field of view. Timing image acquisition to occur during systole also helped to reduce slow flow artefact. Depending on heart rate, each high resolution acquisition took approximately seven minutes. Images were obtained at the level of maximum stenosis and immediately cranial to the stenosis, where the lumen appeared of normal calibre on the cross sectional TOF magnetic resonance angiogram. Total examination time was approximately 40 minutes.

IMAGE ANALYSIS

Conventional digital angiograms (frontal projection) before and immediately after angioplasty were reviewed and per cent diameter stenoses calculated (fig 1). High resolution magnetic resonance images were assessed by two observers reaching a consensus view. Vascular landmarks on conventional and cross sectional TOF magnetic resonance angiograms were used to match images for comparative measurements. Using commercial software to analyse the magnetic resonance images (Advantage Windows, GE Medical Systems), per cent lumenal diameter stenoses were measured at the same levels as on conventional angiography. Coronal measurements were used on transaxial images to allow comparison with coronal diameters obtained on conventional angiography. Per cent cross sectional area of the vessel occupied by plaque was calculated using the formula:

(total vessel area - lumenal area / total vessel area) $\times 100$.

After the initial examination, internal features within the transaxial images were used to match levels at each time point (fig 2). Cine phase contrast flow measurements were made using semiautomated software developed in-house. ${ }^{11}$

\section{Results}

In all patients, the arterial wall was visible but chemical shift and incomplete saturation of flowing blood caused minor artefact. The thickened atheromatous wall and narrowed vessel lumen were clearly identified and in all four cases there was a close relation between conventional angiography and transaxial measurements of per cent diameter stenosis (table 1). As expected, both conventional angiography and magnetic resonance angiography underestimated the extent of disease, in that plaque cross sectional areas in segments of vessels considered angiographically "normal" were significant, ranging from $49 \%$ to $76 \%$ of potential lumenal area. Thus in all four patients extensive atherosclerotic plaques were accommodated by substantial vessel wall remodelling.

Focal variations in plaque signal intensity were seen. However, in the absence of histology, it is not possible to determine whether or not these represented true variations in plaque composition, although this has been previously reported. ${ }^{8}$ Following angioplasty, plaque fissuring and local dissection were easily identified (fig 2). In three patients, angioplasty was 

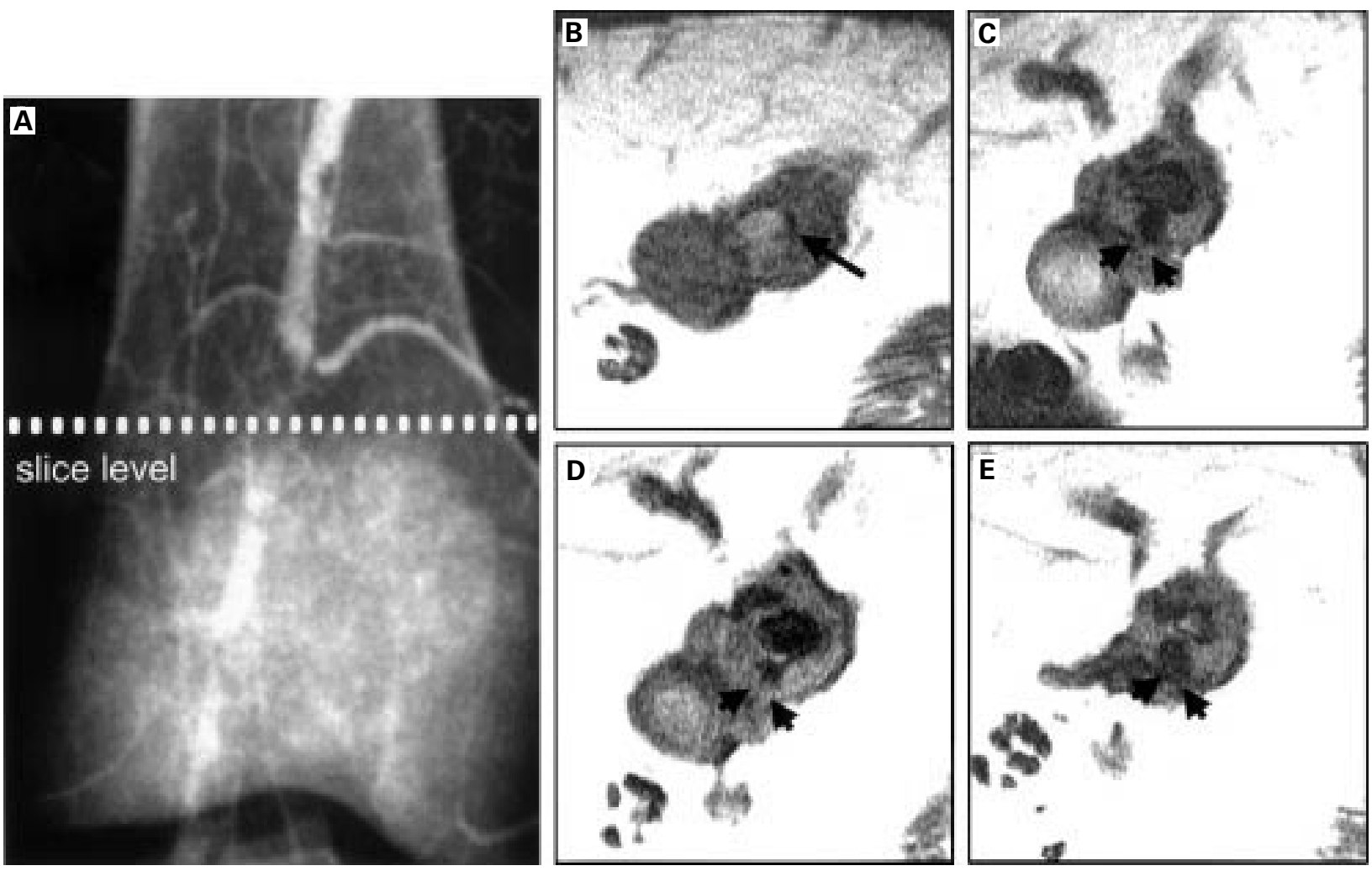

$\mathrm{F}$

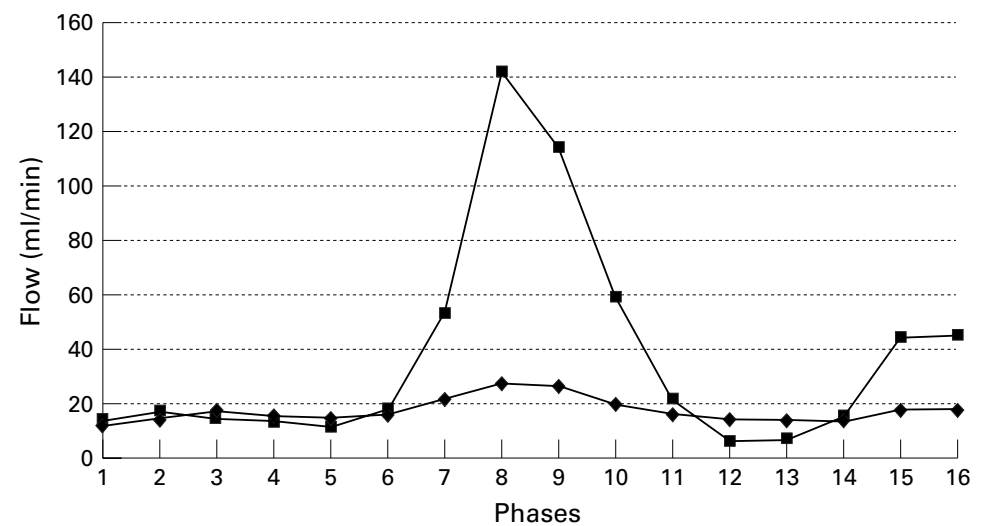

associated with early improvements in vessel lumen diameter and blood flow measured by cine phase contrast acquisition, although a degree of restenosis was subsequently seen in all three. In patient 4, angioplasty to an eccentric plaque failed to produce a significant early improvement in lumen diameter, presumably because of vessel recoil. However, despite this, lumen diameters at three and six months increased, indicating favourable vessel remodelling despite a poor initial result.

\section{Discussion}

The results presented confirm that noninvasive MRI can provide equivalent information to conventional contrast angiography with respect to the lumenal diameter of the popliteal artery. More importantly, it can define the extent of the initial atherosclerotic
Figure 2 (A) Conventional angiogram of the right popliteal artery in patient 1 with a 90\% stenosis, who subsequently underwent angioplasty. High resolution magnetic resonance images at the level of the stenosis show cross sections of the artery before angioplasty (B), at one week (C), at one month (D), and at three months (E). The vessel lumen appears black on all images and is shown as a small slit

preangioplasty (long arrow). Angioplasty produces extensive fissuring which heals with restenosis but fissuring remains (arrowheads $C-E$ ). (F) Flow (ml/min) during 16 phases of the cardiac cycle cranial to the stenosis before angioplasty ( and at one week after angioplasty (⿴).

lesion, the blood flow across it, the damage caused by the angioplasty balloon, and the remodelling process leading to restenosis. The magnetic resonance images confirm in vivo what pathologists have long determined postmortem: atherosclerotic lesions are not homogeneous ${ }^{12}$ and large atherosclerotic lesions can be accommodated within a vessel without necessarily compromising flow or being angiographically significant. ${ }^{5}$

The popliteal artery was chosen because of its accessibility, size, and relative lack of motion, all of which make it highly suitable for MRI. Fuster's group has recently used similar techniques to image human carotid lesions before surgery, ${ }^{9}$ showing that, even with today's technology, the carotid artery is also amenable to MRI. Significant improvements in technology will be needed to obtain similar images in 
Table 1 Popliteal artery atherosclerosis measured by lumen diameter and plaque cross sectional area before and after angioplasty

\begin{tabular}{|c|c|c|c|c|c|c|c|c|c|c|c|c|}
\hline & \multicolumn{3}{|l|}{ Patient 1} & \multicolumn{3}{|l|}{ Patient 2} & \multicolumn{3}{|l|}{ Patient 3} & \multicolumn{3}{|l|}{ Patient 4} \\
\hline & $\begin{array}{l}\text { Angio } \\
(\% \text { dia })\end{array}$ & $\begin{array}{l}M R \\
(\% \text { dia })\end{array}$ & $\begin{array}{l}M R \\
\text { (\% area) }\end{array}$ & $\begin{array}{l}\text { Angio } \\
(\% \text { dia })\end{array}$ & $\begin{array}{l}\text { MR } \\
(\% \text { dia) }\end{array}$ & $\begin{array}{l}M R \\
\text { (\% area) }\end{array}$ & $\begin{array}{l}\text { Angio } \\
\text { (\% dia) }\end{array}$ & $\begin{array}{l}M R \\
(\% \text { dia) }\end{array}$ & $\begin{array}{l}M R \\
\text { (\% area) }\end{array}$ & $\begin{array}{l}\text { Angio } \\
(\% \text { dia })\end{array}$ & $\begin{array}{l}M R \\
(\% \text { dia })\end{array}$ & $\begin{array}{l}M R \\
(\% \text { area })\end{array}$ \\
\hline Preangioplasty & 90 & 85 & 97 & 95 & 91 & 96 & 80 & 83 & 96 & 40 & 50 & 91 \\
\hline One week & 20 & 37 & 72 & 20 & 36 & 69 & 30 & 38 & 60 & 20 & 75 & 93 \\
\hline One month & & 33 & 67 & & 63 & 83 & & 56 & 75 & & 75 & 92 \\
\hline Three months & & 44 & 74 & & 60 & 85 & & 62 & 79 & & 58 & 75 \\
\hline Six months & & 50 & 82 & & 60 & 81 & & 58 & 83 & & 50 & 77 \\
\hline
\end{tabular}

Angio (\% dia), per cent lumen diameter narrowed, assessed by conventional angiography; MR (\% dia), per cent lumen diameter narrowed, assessed by magnetic resonance angiography; \% area, percentage of the total cross sectional area of the artery occupied by plaque as defined by magnetic resonance imaging.

smaller, more mobile vessels, such as the coronary arteries. Better resolution will be required and cardiac and respiratory motion correction must be improved to permit submillimetre accuracy. The position of the coronary arteries deep within the chest makes use of current surface coils impracticable for all but the proximal right coronary artery, and endovascular coils may be needed. ${ }^{12}$ Nevertheless, our results suggest that, with appropriate development and refinement, similar images may ultimately be obtainable in the cerebral and coronary circulations.

The ability to image the same artery repeatedly, with minimal inconvenience and risk to the patient, offers enormous potential both for the surveillance and audit of interventional procedures and for research to define the effects of treatment on the progression of disease. For example, it was long considered that restenosis following angioplasty was caused entirely by smooth muscle cell hyperplasia leading to intimal thickening at the expense of the vessel lumen. This notion spawned a massive (and so far fruitless) investment in time and resources to find an antiproliferative agent to prevent restenosis. However, it is now clear that restenosis is the net result of vessel recoil, intimal thickening, and vessel remodelling. Our study shows that MRI is able to define these pathological consequences of balloon angioplasty as never before, and that important information can be gained from studying the peripheral circulation.

We often found heterogeneity of signal intensity within individual plaques. The significance of this remains to be established, as we were unable to examine the plaques histologically and artefacts are easily generated. However, studies in cholesterol fed rabbits suggest that MRI can discriminate between different components of atherosclerotic plaque and Libby's group have recently reported that MRI can demonstrate sequential qualitative changes in plaque composition which occur after lipid lowering. ${ }^{7}$ In man, early reports comparing magnetic resonance images of carotid atheroma in vivo and histology of the excised lesion suggested that T2 imaging may be able to discriminate plaque composition. These exciting observations herald the prospect of non-invasive longitudinal studies in man to define the effects of lipid lowering or other treatments on plaque morphology.

Our small study shows unequivocally the superiority of MRI over conventional angiography in defining the pathology of atherosclerosis and restenosis in the peripheral circulation and argues strongly for increased research and development to refine the technology and extend its application to other vascular beds.

We acknowledge the support of Addenbrooke's Hospital Cancer Scanner Appeal Fund. PLW is British Heart Foundation Professor of Cardiovascular Medicine.

1 Libby P. Molecular basis of acute coronary syndromes. Circulation 1995;91:2844-50.

2 Davies MJ, Richardson PD, Wolf N, et al. Risk of thrombosis in human atherosclerotic plaques: role of extracellular lipid, macrophage and smooth muscle cell content. $\mathrm{Br}$ Heart f 1993;69:377-81.

3 Weissberg PL, Clesham GJ, Bennett MR. Is vascular smooth muscle cell proliferation beneficial? Lancet 1996; 347:305-7.

4 Hackett D, Davies G, Maseri A. Pre-existing coronary stenoses in patients with first myocardial infarction are not necessarily severe. Eur Heart f 1988;9:1317-23.

5 Glagov S, Weisenberg E, Zarins C, et al. Compensatory enlargement of human atherosclerotic coronary arteries. $N$ Engl f Med 1987;316:1371-5.

6 Brown GB, Zhao X-Q, Sacco DE, et al. Lipid lowering and plaque regression. New insights into prevention of plaque disruption and clinical events in coronary disease. Circulation 1993;87:1781-91

7 Skinner MP, Yuan C, Mitsumori L, et al. Serial magnetic resonance imaging of experimental atherosclerosis detects lesion fine structure, progression and complications in vivo. Nature Med 1995;1:69-73.

8 McConnell MV, Aikawa M, Maier S, et al. High resolution MRI detects rabbit atherosclerosis progression and regression in vivo. Circulation 1997;96:I-191.

9 Troiussant J-F, LaMuraglia GM, Southern JF, et al. Magnetic resonance images lipid, fibrous, calcified, hemor-
rhagic, and thrombotic components of human atherosclerosis in vivo. Circulation 1996;94:932-8.

10 Moss HA, Lomas DJ, Graves MJ, et al. High resolution MRI of the popliteal artery in normal subjects and patients with atheroma [abstract]. Heart 1997;77(suppl 1):P14.

11 Hayball MJ, Coulden RA, Lomas DJ. An automated method for venous flow quantitation by magnetic resonance [abstract]. Br f Radiol 1995:68(suppl 1):46.

12 Quick HH, Zimmermann GG, Erhart P, et al. 'In vivo' high resolution intravascular imaging with a single loop receiver coil. Proceedings of the International Society of Magnetic Resonance in Medicine 1997:796. 